\title{
Estado-nación y comunidad societal. Un análisis crítico de los escritos histórico- políticos de Parsons
}

\author{
Nation-state and societal community. A critical analysis of \\ Parsons' historical-political writings
}

Estado-nação e comunidade societária. Uma análise crítica
dos textos histórico-políticos de Parsons

Natalio Pagés*

\section{RESUMEN}

En su última etapa de trabajo, Parsons se embarcó en dos tipos de proyectos. Por un lado, la continuación de su teoría sistemática a través de investigaciones evolucionistas acerca del devenir histórico de las sociedades humanas. Por el otro, el abordaje de problemas específicos de Estados Unidos y su rol en la situación política global. Aunque en ambos casos los análisis responden, de manera más o menos directa, a la lógica de su modelo tetrasistémico o AGIL, los primeros se orientan a mantener un carácter formal y abstracto, mientras los segundos recuperan un anclaje histórico-político del análisis sociológico. En conjunto, pretenden establecer un andamiaje conceptual homogéneo que permita vincular cuestiones analíticas e históricas sin caer en un uso puramente referencial de la sociedad. Sin embargo, los principales comentaristas e investigadores de la obra parsoniana ponen de relieve las dificultades de su sistema para cumplir este objetivo totalizador. Este artículo busca ampliar la discusión respecto de las tensiones entre el nivel "abstracto" e "históricopolítico" de la etapa evolucionista del pensamiento parsoniano, explorando la contraposición de dos tipos diferentes de ingreso al problema de la sociedad: el abordaje del Estado-nación y su noción de comunidad societal.
Palabras clave: comunidad societal, Estado-nación, evolucionismo, Talcott Parsons. 


\begin{abstract}
In his last stage of work, Parsons embarked on two lines of work. First, the continuation of his systematic theory through evolutionary research on the historical development of human societies. Second, the approach to specific problems of the United States and its role in the global political situation. Although the analyses respond more or less directly to the logic of their tetra systemic or AGIL paradigm in both cases, the former is oriented towards maintaining a formal and abstract character. At the same time, the latter recovers a historical-political anchorage of sociological analysis. As a whole, they aim to establish a homogeneous conceptual structure that allows linking analytical and historical issues without falling into a purely referential use of society. However, the leading commentators and researchers of Parsons' work highlight the difficulties of his system in fulfilling this totalizing objective. This article seeks to broaden the discussion of the tensions between the "Abstract" and "historical-political" levels of the evolutionary stage of Parsonian thought by exploring the contrast between two different types of approaches to the problem of society: the nation-state approach and its notion of societal community.
\end{abstract}

\section{RESUMO}

Na última etapa de seu trabalho, Parsons embarcou em dois tipos de projetos. Por um lado, a continuação de sua teoria sistemática através de pesquisas evolucionistas sobre o devir histórico das sociedades humanas. Por outro, a abordagem de problemas específicos dos Estados Unidos e seu papel na situação política mundial. Embora em ambos os casos as análises respondam, de forma mais ou menos direta, à lógica de seu modelo tetrassistêmico ou AGIL, as primeiras são voltadas a manter um caráter formal e abstrato, enquanto as segundas recuperam uma ancoragem histórico-política da análise sociológica. Em conjunto, elas pretendem estabelecer um arcabouço conceitual homogêneo que permita vincular questões analíticas e históricas sem cair em um uso puramente referencial da sociedade. Contudo, os principais comentaristas e pesquisadores da obra parsoniana destacam as dificuldades de seu sistema para cumprir este objetivo totalizador. Este artigo procura ampliar a discussão sobre as tensões entre os níveis abstrato e histórico-político da etapa evolucionista do pensamento parsoniano, explorando a contraposição de dois tipos diferentes de tratamento do problema da sociedade: a abordagem do Estado-nação e sua noção de comunidade societária.
Keywords: societal community, nation-state, evolutionism, Talcott Parsons.

Palavras-chave: comunidade societária, Estado-nação, evolucionismo, Talcott Parsons. 


\section{Introducción}

En la última etapa de la obra de Talcott Parsons se hallan dos aportes fundamentales a la teoría sociológica. En primer lugar, la elaboración del primer concepto sistemático de sociedad a través de una triple definición que distingue "sistema social", "sociedad moderna" y “Estado-nación”. Un abordaje diferenciado en niveles que permite vincular cuestiones analíticas e históricas sin caer en un uso puramente referencial de la sociedad (Chernilo, 2004). En segunda instancia, la delimitación histórica de los componentes normativos de la sociedad a través de su noción de "comunidad societal".

A la par del concepto de sociedad, fundante de la disciplina, el problema del lazo social fue elaborado significativamente por los autores clásicos de la sociología a través de la noción de "comunidad" ${ }^{1}$. A diferencia de sus antecesores en el análisis de la dimensión adscriptiva de las relaciones sociales, Parsons no limita la comunidad a una antesala histórica de la sociedad moderna ni a una definición de relaciones ideal-típicas de cohesión (de Marinis, 2011). Su análisis intenta superar la dicotomía clásica entre comunidad y sociedad a partir de una recuperación del legado teórico de Durkheim² ${ }^{2}$ Para ello, configura un esquema descriptivo-analítico general donde el sustrato común de solidaridad es una de las funciones constitutivas del sistema social (junto con la asignación de recursos, el logro de metas y el mantenimiento de patrones normativos). La noción de comunidad societal permite analizar los lazos de solidaridad social que son propios de la estructura plural y diferenciada de la sociedad moderna (Parsons, 1971; 1974).

En las últimas décadas, un número importante de investigadores ha revisado esa etapa final de la obra parsoniana, destacando la relevancia y peso potencial de la noción de comunidad societal para la

1 Para un exhaustivo trabajo respecto de la noción de comunidad en la teoría sociológica clásica y algunas de sus derivas contemporáneas, véase Álvaro (2010), Bialakowsky (2010), de Marinis (2010a y 2010b), Grondona (2010), Haidar (2010), Sasín (2010), y Torterola (2010). También el libro Comunidad. Estudios de teoría sociológica, coordinado por Pablo de Marinis (2012b).

2 "Deben evitarse simples dicotomías del tipo de comunidad y sociedad, tan sorprendentemente análogas a la dicotomía entre capitalismo y socialismo. Los intelectuales contemporáneos tienden angustiosamente a retornar a estadios primitivos de comunidad como remedio único para las enfermedades y males de la sociedad contemporánea" (Parsons, 2009, p. 54). 
teoría social (Alexander, 2001; Gerdhardt, 2001; 2002; Mouzelis, 1997; 1999; Sciortino, 2004; 2005; 2010; Treviño, 2001). La expansión de textos acerca de esta etapa fue especialmente intensa entre 2007 y 2011, impulsada por la publicación de American society. Toward a theory of the societal community (Parsons, 2007), obra inédita que marcaba el último gran proyecto teórico parsoniano en torno a la sociedad norteamericana, cuyos papeles de trabajo fueron cuidadosamente editados por Giuseppe Sciortino ${ }^{3}$. El descubrimiento de esta obra, nunca publicada, generó una proliferación de textos que desarrollaron elaboradas y fructíferas reposiciones de la noción, destacando su posición dentro de la biografía de trabajo parsoniana, las razones teóricas de su elaboración y sus cualidades promisorias para el análisis de hechos sociales contemporáneos ${ }^{4}$. La principal voluntad que reunió a estos escritos fue hacerle justicia a Parsons como intelectual, resituar su obra en la posición que merece dentro de las ciencias sociales, y desligarlo de las acusaciones de "pensador conservador", "ideólogo del orden" o "teórico sin agentes sociales" ${ }^{5}$. Los dos editores y comentaristas más relevantes de la obra parsoniana discuten

3 Un trabajo de similar carácter al que había realizado Uta Gerdhardt al editar $O n$ national socialism (Parsons, 1993) durante la década previa.

4 Tanto Chernilo (2010) como de Marinis (2012a) señalan la importancia de esta impronta en la revisión teórica de Parsons y acuerdan especialmente en este último aspecto: el valor de la noción de comunidad societal para el análisis de hechos sociopolíticos contemporáneos. Kivisto y Sciortino (2021) parecen haber recogido el guante, dando lugar a los textos "empíricamente orientados" sobre la comunidad societal en sus trabajos recientes sobre "populismo" y "espacio civil" (pp. 287-303).

5 Las dos primeras críticas fueron propias de los llamados "teóricos del conflicto" (Dahrendorf, 1958; Rex, 1970). La tercera fue esbozada, de diversas formas, por la fenomenología social (Schütz, 2003 y la etnometodología de Harold Garfinkel, quien llegó a sugerir que los sujetos de la sociología parsoniana eran cultural dopes: "producen las características estables de la sociedad al actuar en conformidad con las alternativas de acción preestablecidas y legitimas que la cultura común provee" (1967, p. 68, la traducción es propia). El centro de esa crítica fue recuperado por Giddens (1993) en su teoría de la estructuración: "Parsons discierne un paralelo entre la consideración de Weber de la acción y la preocupación de Durkheim por la obligación moral (interiorizada) que aplica para proveer una solución al 'problema del orden de Hobbes' (...) Parsons plantea y procura resolver este 'problema hobbessiano' (...) mediante el axioma de que los 'valores' forman a la vez los componentes motivacionales de la acción y los elementos centrales del consenso universal, que es la condición de la estabilidad social" (p. 96). Posteriormente, también por Bourdieu (2007) en su teoría de la práctica: "Este punto de vista es el que se adopta a partir de las posiciones elevadas de la estructura social desde las cuales el mundo social se da como una representación —en el sentido de la filosofía idealista pero también de la pintura y del teatro- y desde las cuales las prácticas no son otra cosa que papeles teatrales, ejecuciones de partituras o aplicaciones de planes" (p. 85). 
esas lecturas: Gerhardt (2002) reconoce a Parsons como un académico liberal y Sciortino secunda esa perspectiva varias veces, incluso en la conclusión de su artículo más reciente:

La imagen de Parsons como un sociólogo archiconservador (y un propagandista acrítico del american way of life) emergió en 1960 por una confluencia de circunstancias. (...) A partir de 1990, una ola de estudios académicos restauró la perspectiva biográfica sobre Parsons. Se reconoció entonces que su vida y sus actitudes representaban con bastante coherencia las de un liberal defensor del New Deal. Su trabajo también logró un estatus relativamente estable como autor clásico, casi universalmente reconocido. (p. 16) ${ }^{6}$.

Esta justa preocupación por el reconocimiento de la originalidad, multiplicidad y complejidad de la etapa tardía del pensamiento parsoniano, dejó poco espacio para la reevaluación crítica durante esos pocos años de expansión de textos sobre su obra. La revisión de los preconceptos comunes en cuanto a su orientación política y las motivaciones fundamentales de su obra podría haber estimulado revisiones teóricas que, sin necesidad de imponerle un marco ideológico que le resulta ajeno, reconozcan los límites de su teoría sistémica y las tensiones que eso promueve en sus trabajos histórico-políticos?

$6 \quad$ La traducción es propia.

7 Afortunadamente, algunos trabajos se orientaron en esa dirección durante los últimos años. Por ejemplo, Sadrinas (2012a y 2012b) retoma la crítica de Alexander (2005) en cuanto a las contradicciones de la comunidad societal y rastrea con precisión el problema de la tensión entre inclusión y exclusión social. Junto a Nicolás Rubí (Pagés y Rubí, 2012b), se analizaron las perspectivas críticas de Mouzelis y Alexander respecto de la concepción parsoniana del cambio social. Giordano (2015) rastrea con precisión la transformación del papel del cambio social en las distintas etapas de la teoría de Parsons, demostrando que "pierde el carácter residual que exhibía en el primer modelo", donde era tomado como un "dato", para adquirir el estatuto de hecho problemático "mediante la teoría general de la acción" (p. 227). En un artículo posterior, aborda las diversas críticas que se le realizaron a la "indistinción entre integración sistémica e integración social" en el tratamiento parsoniano de la integración (Giordano, 2020). El artículo de Molina y Vedia (2021), al releer la obra de Parsons sin preconceptos de su "conservadurismo" teórico, logra reorientar creativamente los textos "empíricamente orientados" de Parsons hacia ámbitos y preocupaciones poco señalados de su última etapa —su interés por el romanticismo en la tradición cultural occidental, la vida universitaria norteamericana, el ascenso de la contracultura y las revueltas estudiantiles durante la década de 1960 - sin desestimar, por ello, la necesidad de una crítica radical de su teoría del cambio social. 
Aunque Parsons no fue un defensor acrítico del american way of life, como señala acertadamente Sciortino, persistió en la defensa de una perspectiva de la historia de las sociedades humanas que establecía a la sociedad norteamericana de posguerra como referente utópico-normativo. En un trabajo previo, señalamos las tensiones internas que contiene la noción de comunidad societal en su definición más sistemática, derivadas precisamente de los problemas teóricos de su concepción evolucionista del cambio social (Pagés y Rubí, 2012a). Sumado a su vez a una tendencia marcada hacia la indistinción analítica entre niveles teóricos: el particular (histórico y específico) y el universal (transhistórico y abstracto) en su uso de la comunidad societal.

Precisamente, lo que parece ausente en la conceptualización de la comunidad societal es la distinción de niveles que, siguiendo a Chernilo (2010), Parsons desarrolla ejemplarmente para la sociedad y puede rastrearse en sus estudios histórico-políticos sobre el Estado-nación. Ello parece arrastrar consigo una confusión entre los aspectos descriptivos y prescriptivos del análisis social. Si bien en el nivel abstracto y universal de la teoría sistémica la comunidad societal es presentada por Parsons como un concepto analítico-teórico de carácter descriptivo, al acercarnos a sus estudios histórico-empíricos "asume un costado utópico-normativo de primer rango” (de Marinis, 2011, p. 143).

Como señala con precisión Sasín (2012):

La comunidad societal es pensada por Parsons como un concepto transhistórico, apropiado no solo para la comprensión de la sociedad moderna, sino también aplicable a la interpretación de distintas sociedades históricas. En este sentido, la pretensión es la de exponer una elaboración conceptual abstracta, descontextualizada históricamente y desprovista de carga valorativa. Ahora bien, (...) la comunidad, en gran parte de las perspectivas (clásicas y contemporáneas) (...) ha sido y es un valor que refleja un estado ideal, perdido o alcanzable, de las relaciones humanas. Su utilización en el constructo teórico parsoniano, aun especificada con el adjetivo "societal", no puede prescindir de esta trama referencial. (p. 95).

Surgen, entonces, algunos interrogantes básicos: ¿qué tipo de tratamiento del Estado-nación realiza Parsons en sus estudios histórico- 
particulares? ¿Qué sucede, en cambio, con la elaboración teórica de la comunidad societal, utilizada indistintamente en investigaciones de diverso nivel de abstracción? ¿Qué consecuencias teóricas y/o políticas poseen estas diversas formas de análisis? ¿Hasta qué punto, a través de la revisión de los escritos histórico-políticos de Parsons, es posible hallar una superación de los inconvenientes del marco evolucionista del cambio social en su teoría sistemática?

Nos acercaremos a la producción parsoniana, entonces, resaltando las diversas estrategias de su producción intelectual tardía. Por un lado, la continuación de su teoría sistemática a través de ambiciosos trabajos evolucionistas sobre el devenir histórico de las sociedades humanas (Parsons, 1964; 1971; 1974; 1976). Por el otro, el abordaje sociohistórico de problemas específicos de Estados Unidos y su rol en la situación política global (Parsons, 1959; 1965; 1993; 1999). Los trabajos del primer grupo mantienen un carácter formal y abstracto, que caracteriza a su teoría sistemática, mientras el segundo conjunto recupera un anclaje histórico-político del análisis sociológico. Poner a prueba la correspondencia lógica y conceptual entre el nivel teórico-abstracto y el nivel sociológico-empírico de todo el corpus teórico parsoniano es una tarea que excede a este artículo; sin embargo, avanzar en ese sentido traza un camino provechoso para ajustar nuestra percepción respecto de los alcances y limitaciones de su teoría.

En un modelo a escala de tal programa, nos concentraremos aquí en sus textos "empíricamente orientados" - que siguen siendo poco valorados y reconsiderados - para analizar comparativamente el uso que Parsons hace, en un terreno de aplicación concreta de su teoría sistemática, de las nociones de Estado-nación y comunidad societal. Coincidimos con Chernilo (2010), Gerhardt (2002) y Sciortino (2020) cuando destacan que sus textos histórico-políticos habilitan una caracterización más profunda -0 , en todo caso, menos tendenciosade su sociología. Sin embargo, consideramos que muchas de las conclusiones augurantes a las que arriba Chernilo (2010) al evaluar sus estudios referidos al Estado-nación que permitirían aplicar su batería conceptual a sucesos históricos contemporáneos (el neoliberalismo, la globalización, el populismo, las migraciones internacionales), no pueden extenderse sin más a la noción de comunidad societal o al resto 
del cuerpo de trabajo parsoniano. Utilizar la última etapa de producción teórica de Parsons - el esquema AGIL_ para analizar "no solo los años de la expansión capitalista de la posguerra, sino la propia actualidad" requiere reconocer "los aspectos no democráticos, de asimetría de poder político, contenidos en la misma", como señala adecuadamente Cantamutto (2018, p. 34).

Nuestra hoja de ruta se definirá, entonces, por una primera caracterización general de los trabajos históricos de Parsons respecto del Estado-nación, intentando remarcar las potencialidades teóricas y políticas que surgen de su trabajo "empíricamente orientado", así como algunas de sus dificultades conceptuales irresueltas. En la segunda sección, describiremos sintéticamente la noción de comunidad societal y sus dificultades teóricas, haciendo hincapié en la forma en que Parsons implementa la vinculación entre el nivel "abstracto" y el "histórico-político" de su producción. Intentaremos demostrar que, por varias razones, los escritos histórico-políticos de Parsons no presentan una "salida" o "alternativa viable" a las críticas que se le han realizado a la noción. Finalmente, haremos una breve comparación entre ambas vertientes de sus análisis empírico-históricos (Estado-nación y comunidad societal) para dar paso a nuestras apreciaciones finales.

\section{Estudios histórico-políticos (1): Estado-nación}

En un trabajo centrado en desarrollar y analizar las perspectivas teóricas sobre el Estado-nación, Daniel Chernilo (2010) regresa a la sociología parsoniana tomando una salida metodológica poco contemplada: dejar de lado los textos "sistemáticos" y rastrear la concepción del Estado-nación en sus trabajos "empírico-políticos".

Como ya hemos adelantado, Parsons elabora, situado en el ámbito de la "gran teoría", un sistema teórico de carácter formal-abstracto para organizar lógicamente la mirada sociológica de fenómenos sociales de todo tiempo y lugar, así como análisis empíricos de problemáticas histórico-particulares que hacen uso de ese marco general. Su producción intelectual no es equilibrada: la obra parsoniana abunda en elaboración teórica pura. Sin embargo, los estudios empíricos no son un apéndice de segunda clase en relación con su contribución teórica, sino "una parte integral del trabajo de Parsons" (Rocher, 1974, p. 142). Muchas veces, incluso, aparecerán allí importantes avances de 
investigación que serán incorporados, posteriormente, a sus marcos de análisis general ${ }^{8}$.

Chernilo (2010) sostiene que ahondar en ese ámbito de su producción permitirá avanzar, no solo hacia una más profunda y justa evaluación de su obra, sino hacia una "comprensión sustantiva de la política en la modernidad, en general, y del Estado-nación como organización sociopolítica moderna" (p. 83). A través de la indagación de escritos cuya importancia no había sido debidamente reconocida, Chernilo intenta revalorizar la concepción parsoniana del Estado-nación y la modernidad, desestimando las perspectivas críticas más efectivas y resonantes. Para demostrarlo, delimita tres críticas centrales de la obra de Parsons realizadas por autores específicos: el sesgo internalista (Poggi), la ideología conservadora (Dahrendorf) y el nacionalismo metodológico (Giddens).

En la primera crítica se señala la limitación del marco parsoniano a un análisis interno de las tensiones y/o disrupciones del sistema y sus partes constitutivas. Según Poggi (1965), Parsons deja de lado la importancia de las manifestaciones externas al sistema, que ejercen sobre él una ineludible influencia (por ejemplo, la importancia de las relaciones internacionales en el accionar de los estados modernos). Lejos de plantear que esas presiones sean inexistentes para Parsons o que no estén contempladas en su teoría, Poggi señala que los factores externos del cambio social son, de manera regular, subordinados analíticamente a los procesos internos; si influyen, lo hacen solo de manera secundaria, como modificaciones a una orientación del cambio social ya definida mediante factores internos.

En segundo lugar, la crítica de Dahrendorf (1958) se encuentra analíticamente separada en dos partes, una "conceptual" y otra "normativa". La primera significa un rechazo de toda concepción del cambio social que presuponga un consenso universal ideal como punto de partida del análisis. Por ende, según Dahrendorf, ninguna teoría del cambio social puede o debe describir el conflicto como una "disfunción” si quiere realizar un análisis profundo y serio del problema. Ex-

8 Por ejemplo, el paradigma de las cuatro funciones (AGIL) tiene su origen en una serie de estudios empíricos de laboratorio con pequeños grupos de participantes (Parsons, Bales y Shils, 1953, citado en Fox et al., 2005). 
tendiendo este señalamiento, Dahrendorf realiza una crítica "normativa" que indica la existencia de "fuertes implicancias totalitarias en la teoría de Parsons" (Chernilo, 2010, p. 85); una mirada que anticipa la evaluación de la teoría parsoniana como "esencialmente conservadora" (p. 86) a partir de la década de 1960.

En tercer y último lugar, Chernilo señala brevemente las críticas de Giddens $(1973 ; 2011)$ en relación con los problemas del internalismo y el nacionalismo metodológico. Por lo tanto, la presentación de su postura se halla simplificada y vinculada a la de Poggi: el internalismo es un problema de la teoría sociológica que Parsons no creó, pero del cual tampoco logró desprenderse. Por ende, el Estado-nación ha sido comprendido como un sistema "internamente en desarrollo" y los análisis específicos de los procesos históricos han tomado en cuenta lo "externo" al Estado-nación como un "ambiente" al que la sociedad debe adaptarse. En resumidas cuentas, todo tipo de factor externo es así meramente condicional en la progresión de un cambio social preestablecido (Giddens, 1973).

Habiendo identificado estas tres críticas centrales, Chernilo caracteriza el trabajo sociohistórico de Parsons a través de tres ejes centrales: sus investigaciones referidas al fascismo europeo (1993), el macarthismo (1999) y la ampliación de los derechos civiles en Norteamérica (1965). Según Chernilo (2010), estos análisis "empíricamente orientados" permiten rebatir cada una de las posturas críticas presentadas y recuperar parte de la complejidad y dualidad que Parsons otorgaba a la modernidad y al Estado-nación.

Tras La estructura de la acción social (1937), el interés de Parsons era la comprensión de la sociedad empírica de su tiempo que, en los años 30 y principio de los 40 , abarcaba una realidad dual entre el estado totalitario de la Alemania nazi y el estado de bienestar norteamericano, asociado a los lineamientos keynesianas (Gerhardt, 2002). Para Parsons, el surgimiento de los movimientos fascistas dependió centralmente de factores "internos" a la modernidad (instituciones, ideologías, patrones psicológicos de reacción). Ambos modelos (el americano y el alemán) proponían dos tipos de sociedades diametralmente opuestas y colocaban al mundo en medio de una encrucijada histórica: "el todavía bastante vago e imperfectamente cristalizado sistema de ideas del movimiento nacionalsocialista permanece 
en conflicto extremo con aquellos que han tenido la posición dominante en el mundo occidental y se han institucionalizado como parte de su estructura social" (Parsons, 1993, p. 174). Dado que escribía en plena guerra, Parsons (1993) no daba por garantizado el predominio o triunfo de un tipo de sociedad sobre la otra. La seriedad con la que aborda esta cuestión queda clara en la medida en que lo define como un tema que involucra la "supervivencia de la civilización y los valores occidentales" (p. 309).

En ese sentido, resulta justo plantear que la comprensión de Parsons (1993) del tipo democrático de integración social dentro del Estado-nación está asediado por la posibilidad de desarrollos que impidan su consolidación y ciertamente su expansión a diferentes partes del mundo. Por ende, la preocupación de los intelectuales debería estar ligada no solo a los efectos nocivos del fascismo europeo, sino a la inestabilidad interna (tensiones y conflictos) propia de la heterogeneidad cultural norteamericana, que podría derivar en una amenaza real del orden democrático de la sociedad. Es esa preocupación (las tensiones internas que amenazan la estabilidad de la sociedad norteamericana) la que ocupará el centro de sus trabajos sobre el macarthismo (Social strains in America, 1999) y las políticas de inclusión ciudadana (Full citizenship for the Negro American?, de 1965): "las tendencias al nacionalismo agresivo y a la abdicación de responsabilidades, podrían, si se las implementa, inducir a un severo conflicto con nuestros valores" (Parsons, 1999, p. 216). Parsons defiende la idea de la polarización del mundo (democracia-comunismo) y no discute la importancia de pelear por "el orden político estable de un mundo libre" (p. 213), aunque se pregunta hasta qué punto se lo está haciendo debidamente, respetando la verdadera tradición liberal-democrática del Estado-nación moderno. Por ende, el problema de la "lealtad" para él no era defender o no ciertos valores ante la amenaza del comunismo, sino observar la forma en que esa defensa se establecía: definir correctamente el problema y el nivel de la amenaza para no caer en el riesgo de perder la batalla por los valores en el mismo momento en que se los creía estar defendiendo.

El macarthismo, para Parsons, era el resultante de las tensiones propias de un desarrollo industrial rápido y poco sincronizado, y pudo darse a la luz como una reacción basada en el "símbolo" de una doble 
amenaza comunista. Una amenaza externa y, especialmente, una interna: la figura construida del comunismo como el riesgo a perder el estado natural de bienestar que se había conseguido. Parsons relativiza la "realidad" de esa amenaza, sobre todo en el frente interno, pero no el peso que esa amenaza al bienestar, muchas veces magnificada, supuso. En ese sentido, señala específicamente que fue la situación política internacional (polarización de dos modelos) la que permitió que se expresen salidas antidemocráticas a las tensiones propias de "América" y su modernización. En palabras de Parsons (1999):

las tensiones propias de la situación internacional han incidido en una sociedad que está atravesando importantes cambios internos que han sido, en sí mismos, fuentes de tensión, generando el efecto de superposición de un tipo de tensión sobre la otra. (p. 212).

El centro de su trabajo consiste en demostrar: a) una importante diferencia entre el macarthismo y el fascismo europeo (bajo ningún concepto el macarthismo propone una "nueva forma de sociedad"); y b) la necesidad de viabilizar nuevas salidas a los problemas que le toca afrontar a Estados Unidos, en el frente interno y externo, ante la nueva situación mundial polarizada. De forma similar a lo que sugerirá en Full citizenship for the Negro American? (1965) ${ }^{9}$, su estrategia es la profundización - y no el retroceso- de la industrialización, la diferenciación estructural y la generalización de compromisos de valor universalistas e igualitarios. Ante el macarthismo, que erosionaba las libertades civiles que se intentaban defender, era fundamental sostener el proceso de desarrollo en lugar de replegar las fuerzas modernizantes. De manera similar a su lectura del nazismo, como sintetiza Chernilo (2010), "era necesaria más en vez de menos modernidad" (p. 97).

Basándose en la importancia que estos textos le otorgan al orden internacional y sus efectos sobre la sociedad norteamericana, Chernilo (2010) propone una hábil reconstrucción de la propuesta parsoniana: "concebía al Estado-nación como un desarrollo crucial pero no único o necesario de la modernidad occidental" (p. 83) y, además, "incluye sistemáticamente tanto las tendencias internas como las externas que

9 Ya que Parsons introduce aquí la noción de comunidad societal, nos dedicaremos a trabajar este texto en la segunda sección del artículo. 
afectan a cualquier Estado-nación en cualquier coyuntura determinada y, finalmente, que hacen referencia clara a la existencia empírica de conflictos, presiones y tensiones" (p. 84). Sin duda, y este es un argumento que comparten Alexander (1990), Chernillo (2004; 2010), Turner (1999) y, los estudios "empíricamente orientados" de Parsons permiten acceder a una complejidad de análisis que no hallamos en sus textos teóricos sistemáticos, particularmente en lo que refiere a la concepción de la modernidad y sus características ambivalentes, despejando la noción común de la producción parsoniana como "abstracta" o "conservadora".

Aunque concordamos con esta reevaluación general de las potencialidades del trabajo sociopolítico de Parsons, creemos que eso no habilita a pasar por alto la validez de algunas críticas realizadas a su teoría del cambio social. Chernilo (2010) logra despejarlas presuponiendo una comprensión pobre o inadecuada del trabajo de Parsons. En sus propias palabras:

Dahrendorf tergiversa ideológicamente a Parsons cuando acusa a su sociología de utopismo, conservadurismo e incluso totalitarismo. Por su parte, Poggi subvalora conceptualmente a Parsons al desatender sus esfuerzos por considerar conjuntamente los procesos internos y externos. Finalmente, Giddens se equivoca en un sentido sustantivo porque le atribuye su propio nacionalismo metodológico a Parsons y de este modo yerra en su teorización del Estado-nación como el contenedor natural, racional y definitivo de las relaciones sociales modernas. (p. 88).

El trabajo de Parsons sobre el nazismo, el macarthismo y los derechos civiles permite, en base a su reiterada preocupación por definir la orientación normativa de una política basada en el pluralismo y la inclusión, negar la presunción de adscribirlo ideológicamente al conservadurismo norteamericano y, obviamente, a una lógica totalitaria del Estado y sus funciones. Sin embargo, creemos que es importante separar su orientación ideológica explícita de la posibilidad latente de que sus trabajos movilicen, incluso a expensas del propio Parsons, argumentos de tinte conservador. En ese sentido, las críticas centrales no son resueltas por Chernilo; y si reconocemos su sentido teórico, que Dahrendorf lo acuse falazmente de "conservador" o Giddens de promover un análisis "nacionalista", se vuelven proble- 
mas secundarios para la crítica de su modelo del cambio social. En el caso de Dahrendorf y $\operatorname{Rex}^{10}$, la centralidad de su impugnación de la teoría parsoniana es su limitación del conflicto a un problema de incompatibilidades estructurales, como luego retomará Lockwood (1964), y la falta de explicitación del proceso de surgimiento de las normas y su institucionalización. Estos aspectos del cambio social deberían estar ligados unívocamente al problema de los intereses y la distribución del poder, es decir, a la desigualdad social en el proceso de construcción y definición de patrones normativos.

Los escritos sociohistóricos de Parsons permiten reconocer tensiones y conflictos; pero el centro de la crítica de Dahrendorf se refiere al carácter de esas tensiones y no a su ausencia: las tensiones y conflictos en los análisis parsonianos surgen como resultado del proceso de especificación de normas. Incluso en el caso de un texto "empíricamente orientado" como Social strains in America (1999), solo obtienen referencias muy generales. Aunque reconoce que Estados Unidos posee un proceso de estratificación social y "una estructura de clases" (Dahrendorf, 1999, p. 209), no queda claro de qué forma se expresan las tensiones en relación con esa estratificación, ni de qué modo participan los actores (igual o desigualmente) en la definición e instauración del orden normativo. Parsons centra el problema de la tensión en la dificultad de la política de asumir nuevas responsabilidades en el orden económico, ya que esas responsabilidades se oponen a una tradición de gobierno basada en el laissez-faire (1999). Nos referimos, en definitiva, a una limitación analítica del conflicto, confinada a los requerimientos de la integración sistémica y desestima aquellas tensiones asociadas al problema de la integración social (Lockwood, 1964; Mouzelis, 1997) ${ }^{11}$.

Si continuamos esta línea de análisis, la crítica al internalismo que realiza Giddens (2011) no está limitada, como sugiere Chernilo, a un problema de nacionalismo metodológico. Chernilo propone una sugerente lectura pluralista y cosmopolita de los escritos de Parsons: un

10 Véase la primera sección de Pagés y Rubí (2012a) para una revisión y análisis pormenorizado de las críticas a la concepción parsoniana del cambio social.

11 Hacia este problema se extiende también la crítica de Alexander (2005), aunque se enfoca en las posibles implicancias conservadoras de la noción de comunidad societal en la última etapa de la obra parsoniana. 
tipo de análisis histórico-político que contempla factores externos e internos (en el caso del macarthismo), que no supone una dirección unívoca en el desarrollo de la modernidad (en el caso del nazismo) y que involucra la posibilidad de ordenamientos extraestatales en la modernidad (por ejemplo, la especial importancia a las organizaciones y órdenes normativos internacionales). Pero de todas formas el problema que plantea Giddens excede esos factores y se introduce en los inconvenientes evolucionistas del modelo de cambio social que Parsons adopta desde Economy and Societies (1956) en adelante.

Explicitemos el argumento de Chernilo (2010):

Parsons habría estimado como históricamente inexacto, analíticamente insostenible y políticamente erróneo y peligroso considerar al Estado-nación en su forma liberal-democrática como el resultado necesario del desarrollo de la modernidad. Como tipo específico del orden social que Parsons estimaba deseable, el Estado-nación tiene que ser intencionadamente formado, cuidado, defendido y constantemente reinventado. (p. 107).

Se puede sostener que, en la sociología de Parsons, el Estado-nación liberal-democrático no es el "resultado necesario del desarrollo de la modernidad" a partir de dos factores: a) el nazismo es considerado una forma societal interna de la modernidad; b) la modernidad se encuentra en un "estado permanente de crisis de integración producto de sus tensiones sociales más importantes". Por esas razones, Parsons realiza una defensa del Estado-nación, ya que permite proteger "formas pluralistas de vida mediante una integración universalista basada en el estado de derecho" (Chernilo, 2010, p. 108).

Hasta aquí, acordamos con ambas apreciaciones. En comparación con las primeras aproximaciones de la ciencia estadounidense al nazismo - que definían el proceso como la culminación de una degradación moral-psicológica de los líderes nacionalsocialistas, las perversiones y patologías nerviosas de Hitler o la completa sumisión del pueblo alemán en una crisis de socialización que derivó en la barbarie, la tortura y la demencia generalizada ${ }^{12}$-, Parsons se aleja de

12 Hay un número importantísimo de trabajos que comparten esta perspectiva, aún durante el conflicto bélico. Entre otros véase Haffner (2005), Langer (1972), Murray (1943), Merle (1995) y Saussure (1942). Para una crítica sociológica a este tipo de pers- 
los presupuestos naturalistas, delimitando su análisis a factores estrictamente sociales y considerando al nazismo como una amenaza y un peligro a la estabilidad del Estado-nación moderno, pero sin expulsar sus causas de las tensiones propias del proceso modernizador. Mantener vivo el precepto durkhemiano de no aceptar la explicación de lo social por variables externas a lo social ${ }^{13}$ es otra de las riquezas de sus trabajos "empíricamente orientados", como les llamaba Alexander (2005).

Pero es justo aclarar que la crítica giddensiana señala con precisión algunos problemas teóricos del análisis sin olvidar la existencia de estos factores positivos. Su crítica a la noción de "sistema internamente en desarrollo" (Giddens, 2011, p. 27) no refiere, estrictamente, a la inexistencia de conflictos o tensiones (problemas de integración), de factores externos de análisis (política internacional o geopolítica), de estructuras societales divergentes dentro de la modernidad (por ejemplo, el nazismo). En verdad, la crítica de Giddens — que comparte ciertos rasgos con la de Alexander $(1990)^{14}$ - se dirige a un punto nodal de la teoría de Parsons: la definición de una línea normal de desarrollo evolutivo y la reducción de la relevancia de los factores externos a meros condicionantes de esa orientación endógena, pues solo pueden afectar la movilidad del desarrollo refrenándolo, retrocediéndolo o desviándolo (Girola, 2010). Por ende, el problema no se halla en la ausencia de ciertos factores en la teoría parsoniana, sino en el lugar que ocupan y el rol que adquieren dentro su esquema teórico general, incluyendo en este caso el de sus trabajos "empíricos"15.

pectiva sobre el nazismo y su persistencia contemporánea, véase el primer capítulo de Modernidad y Holocausto (Bauman, 2006).

13 "Llegamos por lo tanto a la regla siguiente: la causa determinante de un hecho social debe ser buscada entre los hechos sociales antecedentes, y no entre los estados de la conciencia individual (...) La función de un hecho social debe buscarse siempre en la relación que sostiene con algún fin social" (Durkheim, 2001, p. 164).

14 "Describir meramente a la diferenciación de forma general, la hace parecer un mecanismo automático de equilibrio, algo que ocurre en cualquier momento en que son necesarios ajustes ante el conflicto y las tensiones" (Alexander, 1990, p. 2).

15 Es importante aclarar que la mayoría de las críticas de Giddens se dirigen, particularmente, a las formulaciones teórico-sistémicas de Parsons y al marco evolucionista que comienza a desarrollar desde la década de 1950. Aunque los estudios sobre el nazismo son previos a la sistematización del marco cibernético-evolucionista parsoniano, ya presentan de manera embrionaria algunos de los problemas teóricos de su evolucionismo social. 
El problema de las afirmaciones de Parsons no se halla, por ende, en su caracterización del nazismo sino en los parámetros de análisis que utiliza para alcanzar esa evaluación. En general, Parsons utiliza analogías médicas ("desviación") o económicas ("regresión") para referirse al nacionalsocialismo. Aunque lo considera un producto de la modernidad, solo puede concebirlo como limitación, retroceso o detenimiento de sus "verdaderos" impulsos democráticos, representados por el que considera su caso más desarrollado (Estados Unidos) ${ }^{16}$. En lugar de definir al nazismo como una orientación opuesta o diversa a los parámetros de su propia orientación utópico-normativa, Parsons universaliza su orientación moral como la línea "correcta y normal" del desarrollo de las sociedades modernas (Estados Unidos posee así un carácter de "verdad" transhistórica sobre la dirección última de la modernidad de la que el nazismo, como fenómeno sociohistórico moderno, carece). El problema se transforma, desde su concepción del cambio social, en una cuestión de cuánto se logren acercar o no las estructuras societales a un sistema normativo ideal que se universaliza como propio de la modernidad; riesgo teórico que Giddens identifica correctamente en La constitución de la sociedad (2011), como una "ilusión normativa".

En conclusión, es preciso admitir que los análisis sociohistóricos o empírico-políticos habilitan una evaluación más justa del trabajo parsoniano. Es posible reconocer en ellos la dualidad y complejidad que Parsons otorgaba a la modernidad y al Estado-nación, despejando falaces o simplificadas acusaciones que se le han proferido: "nacionalista", "conservador" o "pensador abstracto". Parsons era, como plantea Turner (1999), un verdadero "modernista" preocupado por los procesos de integración, inclusión social y libertad civil. Sin embargo, la reapreciación y complejización de la mirada sobre las últimas etapas del trabajo parsoniano no deberían llevarnos a desatender las tensiones teóricas y riesgos conceptuales latentes en su perspectiva evolucionista del cambio social.

16 Para una crítica de la perspectiva evolucionista en estos parámetros véase Fanelli (1986) y Girola (2010). 


\section{Estudios histórico-políticos (2): la comunidad societal}

La comunidad societal surge en los últimos tramos de la obra de Parsons, ante su necesidad de explicar la forma en que las sociedades resolvían el problema de la integración frente a las transformaciones internas resultantes de la modernización (Gerhardt, 2002). Parsons la concibe como ese sustrato de solidaridad común que asegura la integración social, respetando la especificidad de las múltiples unidades que componen una sociedad. Otorga entonces un lugar primordial al componente normativo en tanto garante de la cohesión en contextos sociales de creciente diferenciación funcional y pluralismo cultural. Para cumplir esa función, la comunidad societal debe presentar, en tanto núcleo normativo de la sociedad, un contenido lo suficientemente fuerte y motivacionalmente vinculante (de intenso poder de atracción) como para garantizar mínima solidaridad y, al mismo tiempo, lo suficientemente abstracto y universal como para ser apropiado e interiorizado por grupos de lo más diversos (Alexander, 2005).

Dentro de su marco general, el concepto de comunidad societal ingresa en el esquema de las cuatro funciones (AGIL) como el subsistema integrativo del sistema social que permite mantener la estabilidad del orden normativo frente a los procesos de cambio social y aumentar la capacidad adaptativa de la sociedad. Definida a este nivel de abstracción y universalidad, se convierte en una pieza teórica fundamental de la historia evolutiva de las sociedades humanas que elabora Parsons: un rastreo histórico de las características societales que desembocan en la forma institucional acabada de la democracia moderna. El desarrollo evolutivo de las sociedades se establece sobre un creciente proceso de diferenciación institucional que implica la aparición constante de nuevas unidades, cada vez más especializadas. Esta multiplicación de unidades incrementa los componentes identitarios del sistema social. Frente a estas tendencias disruptivas que desencadena la diferenciación, la comunidad societal constituye el núcleo estructural que aglutina las fuerzas integradoras necesarias para sostener una solidaridad común por sobre los particularismos crecientes (Gerhardt, 2002). A este nivel, pasa de ser un concepto ideado para pensar los problemas integrativos propios del mundo moderno a convertirse en "el substrato básico de solidaridad e in- 
tegración de los más diversos sistemas sociales, en las más diversas constelaciones históricas" (de Marinis, 2011, p. 141).

La progresiva abstracción de la noción estuvo ligada a su creciente circunscripción al marco cibernético-evolucionista. Esta perspectiva teórica de Parsons revistió numerosas dificultades teóricas que han sido extensamente señaladas ${ }^{17}$. Por ende, la noción de comunidad societal, en sus distintas formulaciones, se vio particularmente atravesada por las problemáticas conceptuales de su perspectiva evolucionista. Se pueden distinguir, sintéticamente, dos tipos de tensiones internas: a) la coexistencia teórica (no aclarada ni diferenciada) entre un alcance particular (histórico-específico) y universal (transhistórico-abstracto) de la noción; b) la ambigüedad entre un uso descriptivo (analíticoteórico) y prescriptivo (utópico-normativo) de la misma (Pagés y Rubí, 2012a). Sin embargo, la forma que adquieren estas tensiones, así como sus consecuencias teórico-políticas, no son homogéneas ni idénticas en los diversos trabajos de la última etapa intelectual de Parsons.

El desarrollo de una teoría de la comunidad societal fue un largo y disperso proceso de elaboración, distribuido de forma irregular en numerosos textos. Al evaluarlos cronológicamente, resulta evidente que la noción adquirió progresivamente un nivel mayor de abstracción y universalidad $^{18}$. La versión analítico-descriptiva se completa en cinco años de intenso trabajo teórico: La sociedad. Perspectivas evolutivas y comparativas (1974) y The system of modern societies (1971). La última obra disponible al respecto son sus papeles de trabajo — compilados y editados por Sciortino en 2007-: un imponente proyecto sobre la

17 En la primera sección mencionamos las críticas de Lockwood (1964), Dahrendorf (1958) y Giddens (1995). Algunas otras críticas principales son: la tendencia del sistema al equilibrio a través de ajustes estructurales, que implica concebir el proceso de diferenciación como una reacción sistémica espontánea (Alexander, 1990) y a definir una tajante separación entre cambios dentro y cambios del sistema (Fanelli, 1986); la adaptabilidad como una función del sistema con características inmutables a lo largo de la historia (Gouldner, 1973); y la idea de una tendencia evolutiva constante a partir del proceso de diferenciación estructural, que establece una escala histórica creciente de capacidad adaptativa (Granovetter, 1979). Para un resumen de estas críticas véase la primera sección de Pagés y Rubí (2012a) y Pagés y Rubí (2012b).

18 Para una descripción extensa del trabajo parsoniano sobre la comunidad societal véase Gerdhardt (2001), Alexander (2005) y Sciortino (2004; 2005; 2010). Para una descripción de su lugar dentro de la obra parsoniana puede verse Treviño (2001), Gerhardt (2002), Fox, Victor y Bershady (2005). 
comunidad societal estadounidense en el que aún se encontraba trabajando al momento de su muerte en 1979.

Sin embargo, su preocupación por la comunidad no comenzó en esos proyectos de gran envergadura. Los primeros antecedentes de su identificación del "problema comunitario" en la modernidad se hallan algunos años antes, en estudios específicos sobre la estructura normativa y la integración de la sociedad norteamericana. Parsons se aboca, desde fines de la década de 1950, al problema de la comunidad como un conflicto contemporáneo en varios estudios sociohistóricos o "empíricamente orientados". Estos escritos tuvieron un carácter fundacional respecto de su posterior esfuerzo por desarrollar una teoría de la comunidad societal. Nos referimos a los artículos The principal structures of community (1959) y a Full citizenship for the Negro American? (1965) donde definirá y utilizará, por primera vez, el concepto de comunidad societal en relación estrecha con el modelo de ciudadanía de T. H. Marshall y el problema de la inclusión social.

En The principal structures of community (1959), Parsons ensaya una resolución normativa del "problema hobbesiano del orden" a través de la "cuestión comunitaria". El acento está puesto en hallar, a partir de la territorialidad, un sustrato comunitario de la sociedad norteamericana que permita superar la dicotomía clásica gemeinschaftgesellschaft. Parsons no intenta construir un concepto abstracto de aplicación general, sino "entender la comunidad como el aquí y ahora del sustrato activo de solidaridad en la sociedad americana contemporánea" (Gerhardt, 2002, p. 233). Caracteriza la noción de una "comunidad articulada territorialmente" como "aquel aspecto de la estructura de los sistemas sociales que se refiere a la locación territorial de las personas (...) y sus actividades" (Parsons, 1959, pp. 152-153). La realidad geográfico-histórica estadounidense se asume como punto de partida legítimo para organizar las orientaciones individuales hacia un sentido común de pertenencia normativa dentro de un marco de diferenciación estructural-funcional avanzada. En conclusión, Parsons concibe que el anclaje territorial de cuatro aspectos institucionales (la "ubicación residencial", la "ocupación laboral", la "jurisdicción" y el "complejo comunicativo") regulan la identificación de los grupos con el orden normativo nacional y asegura la integración de la sociedad (Parsons, 1959, p. 163). 
Parsons avanza en la conceptualización de la comunidad societal en el artículo Full citizenship for the Negro American? (Gerhardt, 2001). Recuperando la concepción de triple ciudadanía de T. H. Marshall, intenta perfilar una primera definición de la noción que destaque su carácter inclusivo: "es aquel aspecto del todo social como un sistema que forma una gemeinschaft, que es foco de solidaridad o lealtad mutua de sus miembros y que constituye la base consensual mutua que subyace a su integración política" (Parsons, 1965, p. 423). En ambos escritos, la comunidad societal adquiere, además, un fuerte sentido utópiconormativo (de Marinis, 2011):

Es el foco de lealtades que no necesita ser absoluto, además no lo puede ser, pero que requiere alta prioridad entre las lealtades de los miembros. Para ocupar esta posición, la estructura de asociación debe estar en acuerdo con los valores comunes de la sociedad: los miembros están comprometidos a ella porque esta implementa sus valores y organiza sus intereses en relación con otros intereses. (Parsons, 1965, p. 424).

A partir de la caracterización de los rasgos integrativos y la estructura de solidaridad social de la sociedad estadounidense de posguerra, Parsons identifica el carácter de un proyecto moderno aún incompleto. La prioridad normativa de esta formulación sugiere, enfáticamente, la necesidad de extender y fortalecer un foco común de integración de la sociedad moderna que permita acentuar la inclusión social. En efecto, el análisis de la comunidad societal en este escrito tiene un objetivo político concreto: allanar el camino hacia la inclusión completa de los "americanos negros" en la ciudadanía nacional, observando cómo se comporta el núcleo integrado de la sociedad "americana" ante procesos de cambio en la estructura sociocultural.

De manera similar a su trabajo empírico sobre el Estado-nación, los estudios "comunitarios" exhiben una lectura sutil de las tensiones propias de la modernidad e incorporan al análisis referencias comunitarias materiales y territoriales, un origen particular de las normas, desigualdades históricas entre grupos culturales diversos, conflictos de poder y un reconocimiento explícito de la exclusión socioeconómica. En efecto, configuran un diagnóstico sobre problemas en el orden de las sociedades modernas que no remiten, de manera directa, a incompatibilidades estructurales autorreguladas por el sistema. A 
diferencia de trabajos ulteriores, limitados a la integración sistémica de elementos normativos, Parsons se preocupa aquí por la integración en tanto "inclusión de grupos", en base a una mayor "igualdad de oportunidades", y reconoce la importante existencia conflictiva de grupos sociales enfrentados.

Por esa razón, la problematización del "núcleo" [core] es tan relevante en el caso de Full citizenship for the Negro American? Cuando Parsons se refiere a la organización de la comunidad estadounidense, habla de un estrato poblacional históricamente diferenciado (WASP) ${ }^{19}$ que sostiene los valores centrales y lleva adelante las decisiones gubernamentales en Estados Unidos. El proceso de integración refiere aquí, en parte, a las luchas por los derechos civiles que posibilitan la ampliación de ese "núcleo". Esta es una forma inusual de concebir los conflictos y las tensiones en su obra. Como señala Cantamutto (2018):

expresa justamente lo que en otras corrientes de pensamiento se conoce como construcción de hegemonía, esto es, la prevalencia de un conjunto particular de valores y creencias sobre el conjunto de la población. La construcción de consenso requerida por el proceso democrático de legitimación involucra crear un sentido común, una tradición que guíe la acción en ciertos sentidos. (p. 30).

Si aceptamos la propia lectura sociohistórica que realiza Parsons, la definición de pautas y normas de la "estructura normativa o legal" de la sociedad debería ser asociada a un proceso desigual, basado en la existencia de un "nucleo societal" [core], con poder diferenciado a la hora de definir el contenido de las pautas y la orientación de los valores, y no únicamente a un problema de adecuación sistémica de patrones normativos producidos por la creciente diferenciación estructural ${ }^{20}$. Una perspectiva que el propio Parsons abandonará prontamente.

19 La sigla WASP significa White Anglo-Saxon Protestant, es decir, blanco anglosajón protestante.

20 Cantamutto (2018) suma a este problema el de la coerción, es decir, la forma en que este núcleo sostiene su capacidad diferencial de establecer pautas, patrones y valores que guíen la acción: "Parsons señaló, contra el análisis marxista, que suponer el monopolio de un grupo en la posición de membrecía real es una dicotomía excesivamente simplificada, pero no pudo ofrecer razones teóricas por las cuales esta posición monopólica o cuasimonopólica no podría sostenerse: ¿qué evita que el grupo constituyente original no aproveche su posición privilegiada para contener, administrar o dosificar el acceso de otros grupos? ¿Qué evita que utilice el recurso coercitivo? (...) La falta de re- 
Por ello, la revisión de Alexander (2005) a la comunidad societal plantea que Parsons termina resignando la justicia por la solidaridad. Una comprensión profunda de esta crítica requiere una apreciación del corrimiento conceptual de core, paralelo al proceso de abstracción evolucionista de la teoría parsoniana, que pasa de significar "colectivo social predominante o hegemónico" (en sus escritos histórico-políticos) a "estructura normativa o legal" de la sociedad (en sus escritos teórico-abstractos). Parsons pierde progresivamente su interés en los procesos de integración social y se limita a problemáticas de integración sistémica, confundiendo "integración normativa” con "justicia social" u "orden social equitativo", y presuponiendo erradamente que un aspecto deriva inequívocamente del otro.

En gran parte, se debe a las ideas centrales de su paradigma evolucionista, que tiende a analizar el cambio, el conflicto y la discontinuidad como aspectos de segunda instancia, disrupciones o amenazas a una estabilidad social analíticamente presupuesta (Pagés y Rubí, 2012a). En Societies (1974), una de sus obras cruciales, Parsons afirma, en medio de la descripción de las relaciones entre la sociedad y sus ambientes, que la creciente diferenciación entre los mecanismos operativos (la organización burocrática o los mercados económicos) y las bases culturales de legitimación es una "tendencia del proceso evolutivo" (p. 25) de las sociedades. De la misma forma, asegura que todas las sociedades han tenido (de formas ligeramente variables) núcleos familiares que se encargan de la moralización y constitución de "buenos ciudadanos que se atienen a las reglas" y de instituciones terapéuticas que regulan y mantienen la conducta normal, "respetuosa de los valores y las normas" (pp. 27-30). Es difícil negar la existencia de múltiples relaciones de parentesco o rituales mágico-religiosos en casi todas las sociedades humanas, pero Parsons tiende a inmovilizar históricamente la significación social de esas prácticas y esas instituciones desde una mirada retrospectiva que toma a la concepción moderna del Estado y sus instituciones como "punto de llegada", y vuelve a toda forma social previa una prueba piloto de las instituciones del Estado-nación norteamericano de posguerra.

conocimiento de Parsons sobre esta tensión no es una ingenuidad: en caso de afirmarla, habría caído su ilusión democrática y pluralista de la sociedad (estadounidense) moderna" (pp. 31-33). 
Sin lugar a dudas, como apunta la mayoría de sus comentaristas, los análisis histórico-políticos de Parsons eluden el esquematismo y la rigidez teórica que posee la noción de comunidad societal en sus trabajos evolucionistas, donde la integración de nuevos patrones normativos en base al núcleo ya estable de solidaridad común deja poco margen para cualquier otra forma de transformación, dislocación o disrupción que se aleje de la resolución sistémica "normal" de tensiones y/o conflictos sociales ${ }^{21}$. Sin embargo, la idealización del estándar cultural "americano" (Chernilo, 2004; Girola, 2010) y la concepción de un equilibrio móvil en el progreso de las sociedades (Fanelli, 1986) ha llevado a Parsons a suponer un proceso que uniría de forma lineal y progresiva la diferenciación con la integración, la inclusión y la justicia social.

Molina y Vedia (2021) lo señalan con precisión:

las transformaciones históricas se ubican en una línea de desarrollo continuo y acumulativo. Si el cambio no altera el sistema de valores de la sociedad, será interpretado como progresivo y, en virtud de una confusión entre los niveles descriptivo y prescriptivo, juzgado positivamente. (...) Parsons daba por supuesto que su país estaba a punto de resolver definitivamente el conflicto entre integración y justicia. La ampliación de la ciudadanía creía, uniría el pluralismo con la cohesión social. (pp. 375-376).

Esa linealidad no conflictiva, propia de su teoría sistemática evolucionista, limita los aspectos más promisorios de sus trabajos históricoempíricos sobre la comunidad societal: la identificación de procesos de exclusión y desigualdad en la formulación social de los patrones normativos.

\section{Conclusión}

El trabajo que presentamos contiene la dificultad de reunir la revisión de textos clásicos de Parsons y sus comentaristas contemporáneos, con la relectura precisa de sus textos menos evaluados (aquellos "empíricamente orientados") y nuestras propias apreciaciones críticas. Intentamos hacerlo sin caer en una falsa categorización, simplificación

21 Nos referimos específicamente a la sistematización teórica del concepto en La sociedad (1974), Sistemas sociales (1976) y The system of modern societies (1971). 
o demérito del trabajo parsoniano. Intentamos, sin embargo, señalar problemas que podrían extenderse en diversas direcciones: la relación compleja entre sus diversos niveles de análisis, la comparativa entre el desarrollo de sus distintos modelos de trabajo, los conflictos teóricos crecientes en su teoría evolucionista del cambio social. Dentro de los múltiples caminos posibles, nos propusimos aquí un objetivo específico que esperamos haber delineado claramente: evaluar las potencialidades y dificultades que presentan los estudios histórico-políticos de Parsons (sobre el nazismo, el macarthismo, los derechos civiles y la situación internacional de posguerra) en dos líneas específicas de su trabajo: la pregunta por la sociedad (Estado-nación) y la pregunta por la comunidad (comunidad societal).

En el primer apartado, desarrollamos las características centrales de sus estudios sobre el Estado-nación, analizamos la relectura que realiza Chernilo (2010) y aceptamos buena parte de las potencialidades que reconoce en los trabajos "empíricamente orientados" de Parsons. Sin embargo, aunque promueven efectivamente una representación más justa de su teoría, señalamos la persistencia de inconvenientes teórico-políticos que se mantienen irresueltos. En el segundo apartado, procuramos extender algunas de estas preocupaciones hacia el desarrollo parsoniano de la comunidad societal. Esta noción nos permitió ahondar en conflictos ya señalados durante la primera sección, así como revalorar sus escritos empíricos y evaluar las dificultades que asume la dinámica implícita entre niveles de análisis ("teórico-abstracto" e "histórico-particular") dentro del marco evolutivo que Parsons adopta desde mediados de la década de 1950.

Tanto la comunidad societal como el Estado-nación implican densas conceptualizaciones que permiten explorar el complejo diálogo entre proposiciones formal-abstractas y teórico-empíricas en la sociología de Parsons, uno de los factores característicos de su obra. Sin embargo, el diálogo entre planos analíticos asume formas diferentes en el desarrollo de cada una de las nociones, que no radican en problemas de orden nominal sino en presupuestos teóricos de fondo. Como plantemos al comienzo, el Estado-nación forma parte de un esfuerzo de definición que Parsons realiza respecto a la noción de sociedad, proporcionando categorías adecuadas a distintos niveles de análisis según una gradiente de mayor a menor abstracción 
(sistema social, sociedad moderna, Estado-nación). Por el contrario, este tipo de metodología está ausente en la conceptualización de la comunidad societal. En contraste al elevado nivel de sistematización que supuso el estudio de la sociedad, el desarrollo de la noción de comunidad societal surge de un trabajo fragmentario que mixtura múltiples objetivos y niveles teóricos.

La definición de Estado-nación que se desprende de sus estudios "empíricos" supone una "dinámica teórica" (o marco epistemológico) que no tiene correlato en la noción de comunidad societal. La definición abstracta incorpora, por un lado, una referencia al sistema jurídico-legal como "el foco estructural comparable a la comunidad societal” y, por otro, una enumeración de las unidades sociales modernas en tanto "complejo de redes de lealtad colectiva y colectividades interpenetradas" (Parsons 1971, p. 27). Bajo el esquema evolucionista del cambio social, la estructura y desarrollo de las sociedades tiende a ser definido por un "sentido inmanente" que reduce drásticamente la multiplicidad y la ambivalencia de sus escritos "orientados empíricamente". De esta forma, al definir las características y componentes funcionales de la comunidad societal en tanto subsistema de los sistemas sociales, Parsons se limita a las especificidades de la sociedad occidental moderna (idealizadas, a su vez, a partir del estado de bienestar estadounidense y las políticas keynesianas de la década de 1950) como referente "utópico-normativo".

En forma crecientemente explícita hacia fines de la década de 1960, la existencia de conflictos y contradicciones entre partes diferenciadas de la estructura societal pasan a depender de una instancia preestablecida (mecanismo constituyente de ese mismo sistema) de resolución de tensiones y restitución a un estado de equilibrio. Creemos que es crucial para la teoría social concebir la historia de las sociedades sin la existencia apriorística de factores que determinen la estabilidad o el orden sobre la base de un núcleo de certezas normativas inmóviles. La normatividad del sistema teórico-abstracto de Parsons es, precisamente, aquello que le permite establecer un modelo sistémico de resolución de conflictos que habilita la prevalencia del consenso. Pero el orden normativo consolidado posee un carácter necesariamente hegemónico que Parsons no termina de asimilar teóricamente: extiende como ley colectiva las reglas de juego 
impuestas por ciertos sectores de la sociedad como resultado de pugnas y conflictos que pueden y deben ser analizados en el nivel teórico de la integración social (Lockwood, 1964).

La comunidad societal, en su elaboración más abstracta y general, presenta entonces una serie de tensiones (propias del marco cibernético-evolucionista) que impiden dar cuenta de la dualidad de las relaciones sociales en las sociedades modernas: sus "rasgos histórica, sociológica y normativamente contradictorios" (Chernilo, 2010, p. 12). La forma en que la comunidad societal "debe" asegurar la integración normativa de la sociedad moderna tiene un diseño unívoco (definido en su "proceder" por la lógica cibernético-sistémica y en su "finalidad" por la tendencia evolutiva) que no admite alternativas históricas. Por ende, los rasgos que dan dinamismo, plasticidad y potencia heurística al concepto de Estado-nación se pierden en la producción "comunitaria" del pensamiento parsoniano.

En definitiva, los estudios histórico-políticos de Parsons permiten dar cuenta de factores múltiples y conflictivos en la configuración del cambio social. Sin embargo, el carácter, lugar y función que ocupan en su teoría general, diluyen la complejidad y riqueza que estos aportan a sus investigaciones, pues tienden a ser subsumidos por la lógica idealista de su teoría evolucionista de las sociedades humanas. La consideración de conflictos entre grupos sociales y la historicidad de las disputas por el orden normativo sugieren posibilidades teóricas provechosas para repensar la propia teoría sistémica de Parsons en su nivel formal y general. Se trataría, entonces, de formular principios explicativos que permitan remplazar el universalismo abstracto de la perspectiva evolucionista por un modelo teórico donde la dinámica histórica de los conflictos sociales sea parte del diseño analítico y no una disfunción que se aleja de patrones ideales o preestablecidos de cambio social.

\section{Referencias}

Alexander, J. (1990). Differentiation theory: Problems and prospects. En J. Alexander y P. Colomy (Eds.), Differentiation theory and social change. Columbia University Press.

Alexander, J. C. (2001). Parsons as a republican critic of industrial society: New understandings of the early writings. En G. Pollini 
y G. Sciortino (Eds.), Parsons' the structure of social action and contemporary debates. FrancoAngeli.

Alexander, J. (2005). Contradictions in the societal community: The promise and disappointment of Parsons concept. En R. Fox, V. Lidz, y J. Harold (Eds.), After Parsons: A theory of social action for the twenty first century. Russell Sage Foundation.

Álvaro, D. (2010). Los conceptos de 'comunidad' y 'sociedad' de Ferdinand Tönnies. Papeles del CEIC, 1(58). https://ojs.ehu.eus/ index.php/papelesCEIC/article/view/12289

Bauman, Z. (2006). Modernidad y holocausto. Sequitur.

Bialakowsky, A. (2010). Comunidad y sentido en la teoría sociológica contemporánea: las propuestas de A. Giddens y J. Habermas. Papeles del CEIC, 1(58). https://ojs.ehu.eus/index.php/ papelesCEIC/article/view/12291

Bourdieu, P. (2007). El sentido práctico. Siglo XXI Editores.

Cantamutto, F. (2018). Génesis y tensiones en la comunidad societal de Parsons. Espiral, Estudios sobre Estado y Sociedad, 71(15), 9-38. https://doi.org/10.32870/espiral.v25i71.6574.g6133

Chernilo, D. (2004). El rol de la "sociedad" como ideal regulativo: Hacia una reconstrucción del concepto de sociedad moderna. Cinta de Moebio. Revista de Epistemología de Ciencias Sociales, (21), 175-188. https://cintademoebio.uchile.cl/index.php/ $\mathrm{CDM} /$ article/view/26120/27419

Chernilo, D. (2010). Nacionalismo y cosmopolitismo. Universidad Diego Portales.

Dahrendorf, R. (1958). Towards a theory of social conflict. Journal of Conflict Resolution, 2(2), 170-183. https://doi.org/10.1177\% 2F002200275800200204

Durkheim, E. (2001). Las reglas del método sociológico. Fondo de Cultura Económica.

de Marinis, P. (2010a). Sociología clásica y comunidad: entre la nostalgia y la utopía (un recorrido por algunos textos de Ferdinand Tönnies). En P. de Marinis, G. Gatti, e I. Irazuzta (Eds.), La comunidad como pretexto: en torno al (re)surgimiento de las solidaridades comunitarias. Editorial Anthropos y UAMIztapalapa.

de Marinis, P. (2010b). La comunidad según Max Weber: desde el tipo ideal de la Vergemeinschaftung hasta la comunidad de los 
combatientes. Papeles del CEIC, 1(58). https://ojs.ehu.eus/ index.php/papelesCEIC/article/view/12301

de Marinis, P. (2011). La teoría sociológica y la comunidad: clásicos y contemporáneos tras las huellas de la "buena sociedad". Entramados y perspectivas, Revista electrónica de la Carrera de Sociología, 1(1), 127-164. https://publicaciones.sociales.uba.ar/index.php/entramadosyperspectivas/article/ view/25

de Marinis, P. (2012a). "La comunidad societal de Talcott Parsons, entre la pretensión científica y el compromiso normativista". En P. de Marinis (Coord.), Comunidad: estudios de teoría sociológica (pp. 231-264). Prometeo.

de Marinis, P. (Coord.) (2012b). Comunidad. Estudios de teoría sociológica. Prometeo.

Fanelli, A. (1986). Talcott Parsons y la teoría del cambio social. Ides.

Fox, R. C., Victor L., y Bershady H. J. (2005). Introduction. En R. Fox, V. Lidz, y J. Harold (Eds.), After Parsons: A theory of social action for the twenty first century. Russell Sage Foundation.

Garfinkel, H. (1967). Studies in Ethnomethodology. Prentice Hall.

Gerhardt, U. (2001). Parsons's analysis of the societal community. En J. Treviño (Ed.), Talcott Parsons today. His Theory and Legacy in Contemporary sociology. Rowman y Littlefield Publishers.

Gerhardt, U. (2002). Talcott Parsons. An intellectual biography. Cambridge University Press.

Giddens, A. (1973). Capitalism and modern social theory. Cambridge University Press.

Giddens, A. (1993). Las nuevas reglas del método sociológico. Amorrortu.

Giddens, A. (2011). La constitución de la sociedad. Bases para la teoría de la estructuración. Amorrortu.

Giordano, P. M. (2015). Avatares del cambio en la teoría general de la acción de Talcott Parsons. Sociológica, 30(85), 197-232. https://www.redalyc.org/pdf/3050/305039898007.pdf

Giordano, P. M. (2020). La noción parsoniana de integración en el horizonte de sus críticas. Astrolabio. Nueva época, (25), 321-347. https://revistas.unc.edu.ar/index.php/astrolabio/article/ view/24578 
Girola, L. (2010). Talcott Parsons: a propósito de la evolución social. Sociológica, 25(72), 169-183. https://www.redalyc.org/ pdf/3050/305026713007.pdf

Gouldner, A. (1973). La crisis de la sociología occidental. Amorrortu.

Granovetter, M. (1979). The Idea of 'advancement' in theories of social evolution and development. The American Journal of Sociology, 85(3), 489-515. https://doi.org/10.1086/227047

Grondona, A. L. (2010). La sociología de Emile Durkheim: ¿una definición "comunitarista" de lo social? Papeles del CEIC, 1(58). https:// ojs.ehu.eus/index.php/papelesCEIC/article/view/12295

Haffner, S. (2005). Alemania: Jekyll y Hyde. 1939, el nazismo visto desde dentro. Editorial Destino.

Haidar, V. (2010). De la disolución a la recreación de la comunidad. Un contrapunto entre Max Weber y François Perroux. $P a$ peles del CEIC, 1(58). https://ojs.ehu.eus/index.php/papelesCEIC/article/view/12293

Kivisto, P. y Sciortino, G. (2021). Is populism the shadow of the civil? En J. C. Alexander, P. Kivisto, y G. Sciortino (Eds.), Populism in the civil sphere (pp. 287-303). Polity

Langer, W. C. (1972). The mind of Adolf Hitler: The secret wartime report. Basic Books.

Lockwood, D. (1964). Social and system integration. En G. K. Zollschan y W. Hirsch (Eds.), Explorations in social change (pp. 370383). Routledge.

Merle, R. (1995). Psicoanálisis de Hitler. Leviatán.

Molina y Vedia, A. (2021). Talcott Parsons y sus sepultureros. Perspectivas sobre romanticismo y contracultura. Trabajo y sociedad, 22(37), 355-378. https://www.redalyc.org/articulo. oa? $\mathrm{id}=387368391020$

Mouzelis, N. (1997). Social and system integration: Lockwood, Habermas, Giddens. Sociology, 31(1), 111-119. https://doi.org/10. 1177\%2F0038038597031001008

Mouzelis, N. (1999). Modernity: A non-European conceptualization. British Journal of Sociology, 50(1), 141-159. https://doi. org/10.1111/j.1468-4446.1999.00141.x

Murray, H. (1943). Analysis of the personality of Adolph Hitler. Donovan Nuremberg Trials Collection from Cornell University Law Library. 
Pagés, N. y Rubí, N. (2012a). Comunidad societal y cambio social: tensiones evolucionistas en la obra de Parsons. En P. de Marinis (Ed.), Comunidad: estudios de teoría sociológica. Prometeo.

Pagés, N. y Rubí, N. (2012b). Mouzelis y Alexander: lecturas críticas del esquema evolucionista de Talcott Parsons. Revista Temas Sociológicos, (16), 37-61. https://doi. org/10.29344/07196458.16.280

Parsons, T. (1959). The principal structures of community: a sociological view. En C. J. Friedrich (ed.), Community (pp. 152-179). Liberal Arts Press.

Parsons, T. (1964). Evolutionary universals in society. American Sociological Review, 29(3), 339-358. https://www.jstor.org/stable/2091479

Parsons, T. (1965). Full citizenship for the Negro American? A sociological problem. Daedalus, 94(4). http://www.jstor.org/ stable/20026954

Parsons, T. (1971). The system of modern societies. Englewood Cliffs.

Parsons, T. (1974). La sociedad. Perspectivas evolutivas y comparativas. Trillas.

Parsons, T. (1976). Sistemas sociales. En D. Sills (Ed.), Enciclopedia Internacional de las Ciencias Sociales. Ediciones Aguilar.

Parsons, T. (1993). On national socialism. Aldine de Gruyter.

Parsons, T. (1999). Social strains in America. En B. S. Turner (Ed.), The Talcott Parsons Reader (pp. 207-220). Blackwell Publishers.

Parsons, T. (2007). American society. Toward a theory of the societal community. Paradigm Publishers.

Parsons, T. (2009). Autobiografía intelectual: elaboración de una teoría del sistema social. Universidad Nacional de Colombia.

Parsons, T., Bales, R. F., y Shils, E. A. (1953). Working papers in the theory of action. Free Press.

Poggi, G. (1965). A main theme of contemporary sociological analysis: Its achievements and limitations. British Journal of Sociology, 16 (4), 283-94.

Rex, J. (1970). Key problems of sociological theory. Routledge y Kegan Paul.

Rocher, G. (1974). Talcott Parsons and American Sociology. Thomas Nelson and Sons. 
Sadrinas, D. (2012a). La comunidad societal en la obra de Talcott Parsons: tensiones entre la inclusión y la exclusión. En P. de Marinis (Ed.), Comunidad: estudios de teoría sociológica (pp. 265-307). Prometeo.

Sadrinas, D. (2012b). El papel de la diferencia en la comunidad societal de Talcott Parsons: la integración a través del conflicto. Entramados y Perspectivas. Revista de la Carrera de Sociología, 2(2), 155-174. https://publicaciones.sociales.uba.ar/index. php/entramadosyperspectivas/article/view/135/119

Sasín, M. (2010). La comunidad estéril. El recurso comunitario como forma de la autodescripción social. Papeles del CEIC, 1(58). https://ojs.ehu.eus/index.php/papelesCEIC/article/ view/12299

Sasín, M. (2012). ¿Por qué le habrá puesto "comunidad"? Una aproximación al lugar de la comunidad societal en la teoría de Talcott Parsons. Sociológica, 27(77), 71-107. http://www.scielo. org.mx/pdf/soc/v27n77/v27n77a3.pdf

Saussure, R. (1942). The psychopathology of Adolf Hitler. Free World.

Schutz, A. (2003). El problema de la realidad social. Escritos I. Amorrortu.

Sciortino, G. (2004). The societal community. Conceptual foundations of a key action theory concept. Kobe University.

Sciortino, G. (2005). How different can we be? Parsons's societal community, pluralism, and the multicultural debate. En R. Fox, V. Lidz, y J. Harold (Eds.), After Parsons: A theory of social action for the twenty first century (pp. 111-135). Russell Sage Foundation.

Sciortino, G. (2010). A single societal community with full citizenship for all: Talcott Parsons, citizenship and modern society. Journal of Classical Sociology, (10), 239-258. https://doi. org/10.1177\%2F1468795X10371715

Sciortino, G. (2020). A blueprint for inclusion: Talcott Parsons, the societal community and the future of universalistic solidarities. The American Sociologist, 52(1), 159-177. https://doi. org/10.1007/s12108-020-09470-0

Torterola, E. (2010). Racionalización y comunicación en la esfera económica. Los matices del individualismo en la teoría de la modernidad weberiana. Papeles del CEIC, 1(58). 
Treviño, J. (2001). Introduction: The theory and legacy of Talcott Parsons. En J. Treviño (Ed.), Talcott Parsons today. His theory and legacy in contemporary sociology. Rowman y Littlefield Publishers.

Turner, B. S. (1999). The contribution of Talcott Parsons to the study of the modernity. En B. S. Turner (Ed.), The Talcott Parsons reader. Blackwell Publishers. 